\title{
Comportamiento materno y bienestar de la coneja doméstica y silvestre y su camada
}

\author{
P. González-Redondo ${ }^{1, *}$, G. González-Mariscal², M. López³, \\ J. Fernández-Carmona ${ }^{4}$, A. Finzi ${ }^{5}$ y A. Villagrá6
}

1 Departamento de Ciencias Agroforestales. Escuela Técnica Superior de Ingeniería Agronómica. Universidad de Sevilla. Sevilla, España

2 Centro de Investigación en Reproducción Animal. CINVESTAV-Universidad Autónoma de Tlaxcala. Tlaxcala, México

3 Departamento de Producción Animal y Ciencia de los Alimentos. Facultad de Veterinaria. Universidad de Zaragoza. Zaragoza, España

4 Departamento de Ciencia Animal. Universidad Politécnica de Valencia. Valencia, España

5 Centro Experimental de Cunicultura Alternativa. Viterbo, Italia

6 Centro de Tecnología Animal CITA-IVIA. Segorbe (Castellón), España

\section{Resumen}

El conejo europeo (Oryctolagus cuniculus), además de tener importancia faunística en el Mediterráneo occidental, es una especie ganadera relevante que es la base de un subsector pecuario industrializado orientado a la producción de carne en varios países, sobre todo europeos, mientras que en algunos países en vías de desarrollo se explota bajo sistemas alternativos orientados a la integración de renta y a la seguridad alimentaria. A la orientación cárnica se suman otras aptitudes productivas heterogéneas que configuran una gran diversidad de sistemas de producción cunícola. Este trabajo revisa el comportamiento materno de la coneja y de su camada, incluyendo su regulación endocrina, tanto en el animal silvestre como en la producción cunícola comercial y alternativa, y se relaciona con los factores de manejo, con la productividad en granja y con el bienestar de la especie. Se analizan también las implicaciones que las normativas sobre bienestar animal comportan respecto al alojamiento, manejo y satisfacción de las necesidades etológicas de las conejas reproductoras y de los gazapos durante la cría, caracterizadas, fundamentalmente, porque en algunos países tienden a proporcionar más espacio y enriquecimiento ambiental en las jaulas.

Palabras clave: Oryctolagus cuniculus, etología, cría, gazapo, endocrinología, alternativas.

\section{Abstract \\ Maternal behaviour and welfare of the domestic and wild rabbit doe and its litter}

The European rabbit (Oryctolagus cuniculus), in addition to its faunal interest in the western Mediterranean, is a relevant species which in several European countries is the basis of a meat-oriented, industrial livestock subsector, while in many developing countries rabbits are raised under alternative systems aimed at income integration and food security. In addition to meat production, other productive ori-

\footnotetext{
* Autor para correspondencia: pedro@us.es
} http://dx.doi.org/10.12706/itea.2015.021 
entations exist that generate a variety of rabbit production systems. This paper reviews the ethology of maternal behaviour of the breeding doe and her litter, including its endocrine regulation, both in wild animal and in industrial and alternative farming systems, and its relation to management factors, productivity and performance as well as the welfare of the species. It also discusses the implications of the regulations concerning animal welfare on housing, management and satisfaction of behavioural needs of breeding does and their litters, which in some countries tend to provide more space and environmental enrichment in cages.

Key words: Oryctolagus cuniculus, ethology, breeding, kit, endocrinology, alternative.

\section{Introducción}

El conejo común (Oryctolagus cuniculus), originario de la Península Ibérica, se domesticó en la Edad Media (Lebas et al., 1996), muy recientemente en comparación con otras especies, por lo que conserva en cautividad parte de sus patrones de comportamiento silvestre (EFSA, 2005), característicos de una especie presa con organización social en colonias que viven en madrigueras. Su explotación ganadera se intensificó más tarde que otros sectores como el porcino y el avícola, experimentando sobre todo una evolución de las técnicas reproductivas (Vega et al., 2012), alcanzando su máxima expresión en países del Mediterráneo occidental (principalmente Italia, España y Francia) con producción y consumo relevantes (Lebas et al., 1996), y expandiéndose a otras áreas del Mundo. Además, gozan de gran difusión la cría de traspatio y para la integración de renta y mejora de la seguridad alimentaria en países en desarrollo (Finzi y Mariani, 2011). Sus diferentes aptitudes productivas (carne, piel, pelo, repoblación cinegética, mascota, razas de fantasía, experimentación) originan sistemas de producción dispares. En este contexto, el repertorio materno de la coneja silvestre y doméstica, controlado hormonalmente, comprende un conjunto de comportamientos de la hembra durante el periparto y la cría de la camada, condicionando el bienestar de ambos y la productividad en granja: excavación de una madri- guera de parto en libertad; introducción en ella de material vegetal seco o, en granja, su acomodación en el nidal; arranque de pelo para forrar el nido; parto en la madriguera o el nidal; y amamantamiento, acompañado ello de una reducida incidencia de conductas que producen pérdidas de gazapos. El objetivo de este trabajo es revisar los resultados científicos sobre conducta materno-filial y sobre la regulación fisiológica del comportamiento maternal y el de su camada durante la cría en conejos silvestres y domésticos, así como analizar su grado de aplicación al manejo y bienestar en la cunicultura comercial y alternativa y su tratamiento en la escasa normativa específica promulgada sobre bienestar en el ámbito europeo. Esta revisión puede ser útil, además, para orientar con base científica la legislación en materia de bienestar de la coneja reproductora y su camada en concordancia con su etología.

\section{Comportamiento materno de la coneja silvestre en libertad}

Excavación de la madriguera de parto, construcción del nido y parto

En libertad las conejas dominantes de la colonia paren en el vivar comunal y las subordinadas, que son mayoría, elaboran el nido y amamantan a sus gazapos en una hura o pequeña madriguera ciega independiente excavada en el terreno lejos del vivar, de hasta 
un metro de longitud, en cuyo fondo se sitúa una cámara nido de unos $25 \mathrm{~cm}$ de diámetro. La hembra la excava e introduce hierba seca dos o tres días antes del parto y no suele volver a entrar hasta pocas horas antes del parto, forrando entonces el nido con pelo de su pecho y abdomen. La coneja bloquea la entrada de la hura con un tapón de unos 10 $\mathrm{cm}$ de suelo que retira cuando entra y repone al salir (Lloyd y McCowan, 1968).

Las hembras silvestres paren por la noche ya que así evitan visitar la hura durante el día y reducen el riesgo de predación (Lloyd y McCowan, 1968). El parto dura unos 10 minutos. A su término, la hembra lame los gazapos, consume la placenta, los amamanta durante menos de un minuto y los abandona cerrando la entrada de la hura. Los gazapos nacen desnudos, con los ojos cerrados y los pabellones auriculares replegados; generan muy escaso calor endógeno y tienen poca coordinación motora (Hudson y Distel, 1982).

\section{Lactación y destete}

El cuidado materno es altamente eficiente pese a que la coneja tiene un limitado contacto con los gazapos durante la lactancia, pues prácticamente no los cuida y los limpia poco. En las dos primeras semanas en que los gazapos están confinados en la hura, la mayoría de las hembras silvestres sólo la visitan una vez al día para amamantarlos (Hudson et al., 1996), principalmente entre 30 y 90 minutos antes de la salida del sol, permaneciendo en el interior unos 15 minutos. El breve amamantamiento una sola vez al día es un mecanismo evolutivamente estable que minimiza el riesgo de predación (Lloyd y McCowan, 1968). Los gazapos abren los ojos a los 10 días de edad (Hudson y Distel, 1982), progresando hacia la homeotermia conforme crecen, de modo que sobreviven en los primeros días gracias a que la temperatura en la hura es prácticamente constante debido a su situación enterrada y al recubrimiento de hierba seca y pelo (Gibb, 1993). Las madres tapan la boca de la hura al menos hasta que los gazapos tienen entre 20 y 22 días (Broekhuizen et al., 1986).

Los gazapos comienzan a salir sobre los 18 días de edad. Al principio muestran un estado de exploración esporádica que dura unas 24 horas durante las que permanecen en la entrada de la hura, pero dos días más tarde ya se desplazan a poca distancia y pastan intermitentemente (Lockley, 1961). El abandono definitivo del nido parece ocurrir a iniciativa de los gazapos (Myers, 1958) cuando tienen unos 23 días, aunque durante varios días después de la dispersión se agrupan en la hura o cerca para mamar (Broekhuizen et al., 1986). El destete es progresivo, de manera que muchos gazapos de más de cuatro semanas de edad pueden estar mamando todavía, sobre todo la última camada de la temporada, disfrutando de una lactancia más larga (Lloyd y McCowan, 1968).

Plasticidad de la etología reproductiva en conejos silvestres

El comportamiento reproductor de los conejos silvestres es dúctil y difiere en función de factores como la disponibilidad de sustrato excavable o la presión de predación. Así, existen poblaciones que no viven estructuradas en colonias familiares en vivares, sino que viven permanentemente en superficie, excavando gazaperas solamente para la cría de las camadas (Kolb, 1991).

\section{Comportamiento materno de la coneja doméstica}

El nidal y el nido en la producción del conejo en cautividad; parto

Pese a la domesticación, la conducta innata de elaboración del nido en conejas domésticas es 
similar a la de las silvestres (Deutsch, 1957). La hura en la que la coneja silvestre hace su nido se sustituye en cunicultura por un nidal cuyo diseño es importante para mantener la temperatura adecuada para los gazapos (Hudson y Distel, 1982; González-Mariscal et al., 1994). Las conejas domésticas aceptan el nidal como lugar para elaborar el nido con el material que el cunicultor introduce, que suele ser paja, viruta de madera o borra, recubriéndolo con pelo que se arrancan del abdomen y pecho. La naturaleza del sustrato introducido en el nidal influye en la calidad del estrato de pelo afectando a la intensidad de la depilación y a la mortalidad de los gazapos durante la lactancia (Verga et al., 1984).

La calidad del nido, medida como la abundancia de pelo y el grado en que cubre los gazapos, se correlaciona positivamente con el número de gazapos destetados. Las camadas que viven en nidos de peor calidad tienen mayor mortalidad y peor crecimiento, sobre todo en los primeros días de edad (Szendrö et al., 1988; Canali et al., 1991). La cantidad y calidad del pelo con el que las conejas domésticas elaboran el nido varía entre razas. Además, con temperaturas ambientales frías tienden a depositar más pelo en el nido (Szendrö et al., 1988). En general, la calidad del nido mejora con el orden de parto (Ross et al., 1956).

\section{Lactancia y destete}

Las conejas domésticas amamantan por primera vez inmediatamente tras el parto, y a partir de entonces normalmente lo hacen regularmente una vez al día durante un tiempo de entre tres y cinco minutos (González-Mariscal et al., 2013a). El amamantamiento ocurre prevalentemente con periodicidad circadiana unas horas antes del inicio del período de luz. En efecto, bajo condiciones de luz:oscuridad controladas dentro de un bioterio de laboratorio (luces encendidas de 07:00 a 21:00 h), alojándose las madres en jaulas grandes con libre acceso a los gazapos, se encontró que la hora de amamantamiento preferida eran las 03:52 (González-Mariscal et al., 2013b). Sin embargo, también se ha descrito que una proporción de conejas sometidas a un fotoperiodo rutinario de 16L:80 amamanta a los gazapos durante las dos primeras horas del periodo de luz (Matics et al., 2012) o las dos primeras del período de oscuridad con 12L:120 (Hoy et al., 2000). La restricción del amamantamiento a una sola vez diaria parece depender de la hembra, pues si los gazapos tienen ocasión intentan mamar más frecuentemente (Zarrow et al., 1965) y, como se verá después, se ha observado que cierta proporción de conejas amamantan más de una vez al día (Hoy et al., 2000). Existe una sincronía circadiana entre la conducta de la hembra y la de los gazapos en el amamantamiento (Hudson et al., 1996). Así, las crías se anticipan a la llegada de la madre de modo que, cuando la coneja entra en el nidal, los gazapos ya se encuentran despiertos y listos para iniciar el amamantamiento. Esta conducta de los gazapos se debe a una sincronización provocada por el alimento, i.e., por la leche recibida en días anteriores (Caba y González-Mariscal, 2009; Morgado et al., 2011). Al colocarse la madre sobre los gazapos adoptando una postura arqueada (cifosis) permite que ellos localicen rápidamente los pezones (Hudson y Distel, 1982) al percibir por vía olfativa la llamada "feromona mamaria", identificada como el compuesto 2-metil-but-2-enal (Schaal et al., 2003). Después de amamantar, la hembra defeca unas pocas heces dentro del nido, pero nunca orina dentro de él. El amamantamiento cesa bruscamente cuando la coneja abandona el nidal y deja a los gazapos solos hasta el día siguiente (González-Mariscal et al., 1994 y 2013b).

El periodo de dependencia maternal se divide en tres etapas (Hudson et al., 1996): i) la primera semana durante la cual los gazapos 
sólo toman leche; ii) las semanas segunda y tercera durante las que comienzan a mordisquear las heces y el material de cama depositado en el nido, hacia el día 11 o 12 de edad, comenzando así a establecerse la flora ceco-cólica en los gazapos, e iniciando el abandono del nidal a partir de los 13 días (Hudson y Distel, 1982), comenzando también a beber agua; y iii) la cuarta semana en la que los gazapos toman cantidades significativas de alimento sólido y agua ya hacia el día 22 ó 23 (Lincoln, 1974).

En cunicultura se practica el destete brusco, separándose comúnmente los gazapos de su madre entre los 28 y 35 días de edad. Se considera un destete próximo al natural porque en ese momento la coneja produce ya poca leche, sobre todo si está gestante simultáneamente (Lincoln, 1974; Hudson et al., 1996). Los destetes más precoces, no difundidos como práctica generalizada aunque se siguen ensayando sus posibilidades de aplicación
(Gómez-Conde et al., 2004; Xiccato et al., 2004), pueden ser más estresantes para los gazapos y comportar más trastornos intestinales, mortalidad y retardo del crecimiento (De Blas et al., 1999).

\section{Mecanismos hormonales que regulan el comportamiento materno de la coneja}

Fluctuaciones hormonales durante la gestación y su relación con la construcción del nido

La concentración sanguínea de varias hormonas cambia a lo largo de la gestación (González-Mariscal et al., 1994), conforme se detalla en la Tabla 1. En general, los niveles de estradiol 17- $\beta$ y de progesterona se incrementan gradualmente a lo largo de la gestación. Sin embargo, al aproximarse el parto la relación entre estas dos hormonas cambia

Tabla 1. Concentraciones hormonales (medias) durante la gestación y la lactancia en la coneja Table 1. Hormone concentrations (means) during pregnancy and lactation in the doe

\begin{tabular}{|c|c|c|c|c|c|c|c|c|c|c|c|}
\hline \multirow{2}{*}{ Hormona } & \multicolumn{4}{|c|}{ Días de gestación } & \multicolumn{7}{|c|}{ Días de lactancia } \\
\hline & 10 & 20 & 25 & 30 & 1 & 3 & 5 & 10 & 20 & 25 & 30 \\
\hline Estradiol ${ }^{1}, \mathrm{pg} / \mathrm{ml}$ & 55 & 65 & 61 & 75 & 63 & 58 & 60 & 64 & 65 & 64 & 57 \\
\hline Progesterona ${ }^{1}, \mathrm{ng} / \mathrm{ml}$ & 7 & 9 & 6 & 3 & - & - & - & - & - & - & - \\
\hline Testosterona ${ }^{1}, \mathrm{pg} / \mathrm{ml}$ & 250 & 260 & 250 & 300 & 200 & 175 & 170 & 180 & 200 & 160 & 140 \\
\hline Prolactina ${ }^{2}, \mathrm{ng} / \mathrm{ml}$ & 20 & 10 & 5 & 170 & nd & nd & 64 & $\begin{array}{c}73 \\
\text { (día 9) }\end{array}$ & 55 & 11 & 10 \\
\hline Oxitocina ${ }^{3}, \mathrm{pg} / \mathrm{ml}$ & nd & nd & nd & nd & $\begin{array}{c}34 \\
\text { (día 2) }\end{array}$ & nd & 87 & $\begin{array}{c}347 \\
\text { (día 11) }\end{array}$ & 105 & nd & 453 \\
\hline
\end{tabular}

nd: no determinada. —: indetectable.

1 Valores tomados de González-Mariscal et al. (1994).

${ }^{2}$ Tomados de McNeilly y Friessen (1978) (valores durante la gestación) o de Fuchs et al. (1984) (valores durante la lactancia, determinados a los 5 minutos después de iniciada la succión).

${ }^{3}$ Valores determinados a los 3 minutos después de iniciada la succión, según Fuchs et al. (1984). 
drásticamente pues el estradiol 17- $\beta$ se eleva bruscamente hacia el día 30 de gestación en tanto que la progesterona disminuye hasta ser prácticamente indetectable el día del parto. Este cambio en la relación estradiol 17$\beta /$ progesterona provoca, a su vez, la brusca secreción de prolactina. La testosterona también se secreta abundantemente durante la gestación, sin mostrar grandes variaciones en su concentración. Todas estas variaciones hormonales (mostradas en detalle en la Tabla 1) son esenciales para regular la construcción del nido maternal, donde tendrá lugar el episodio diario de amamantamiento. La construcción del nido consiste en el despliegue secuencial de tres actividades: 1) la excavación de una madriguera subterránea (en la naturaleza) o de un sustrato colocado dentro de la caja-nido (en el laboratorio o la granja); 2) el acarreo de vegetación comestible del entorno (conejos silvestres) o de paja colocada en la jaula del animal (conejos domésticos) y su introducción dentro de la madriguera o caja nido; 3 ) el arrancado del pelo ventral para recubrir con este material el "nido de paja" construido dentro de la madriguera o caja. El inicio y la terminación de cada una de estas tres actividades están estrechamente regulados por combinaciones hormonales específicas (González-Mariscal et al., 1994). Como se observa en la Figura 1.A, la excavación del sustrato se incrementa conforme se avanza en la gestación, es decir, con altas concentraciones de estradiol 17- $\beta$ y de progesterona y declina hacia final de la misma, cuando disminuyen los niveles de progesterona. Estas variaciones se replican al inyectar a conejas ovariectomizadas benzoato de estradiol 17- $\beta$ y progesterona (González-Mariscal et al., 1996). El acarreo de paja al interior del nido se observa solamente al declinar la concentración sanguínea de progesterona al final de la gestación o al dejar de administrar esta hormona a conejas ovariectomizadas tratadas con benzoato de estradiol 17- $\beta$ (Figura 1.B). El arrancado del pelo se inicia poco antes del parto, coincidiendo con la abrupta secreción de prolactina, el cambio en la tasa estradiol 17- $\beta$ /progesterona y la continua presencia de testosterona. Este comportamiento continúa por varios días post-parto y también se replica en las conejas ovariectomizadas tratadas con benzoato de estradiol 17- $\beta$ + progesterona una vez que se ha removido la progesterona (González-Mariscal et al., 1994 y 1996). La implantación de benzoato de estradiol 17- $\beta$ en el área preóptica del cerebro estimula la excavación y el acarreo de paja en conejas ovariectomizadas, cuando se acompaña de la inyección subcutánea de progesterona (y posterior remoción; González-Mariscal et al., 2005). La prolactina también participa en regular la construcción del nido maternal pues: a) la inyección subcutánea de bromocriptina (droga que antagoniza la liberación de prolactina) en los últimos días de la gestación previene el acarreo de paja y el arrancado del pelo (González-Mariscal et al., 2000), y b) la inyección de prolactina por vía intracerebroventricular revierte los efectos provocados por la bromocriptina (González-Mariscal et al., 2004).

Relevancia de las hormonas de la lactancia para la expresión del comportamiento maternal

Las concentraciones sanguíneas de estradiol $17-\beta$, progesterona, testosterona y prolactina observadas durante la gestación cambian drásticamente durante la lactancia (ver Tabla 1). Además, las dos hormonas clásicas de la lactancia, es decir, la prolactina y la oxitocina muestran una liberación fásica y breve. En cada episodio de succión, que dura entre 3 y 5 minutos (González-Mariscal et al., 1994), y ocurre sólo una vez cada 24 horas dentro del nido (Jilge, 1993 y 1995; González-Mariscal et al., 2013b), se observa la secreción máxima de oxitocina y prolactina (Tabla 1). Sin embargo, el brusco incremento en la con- 


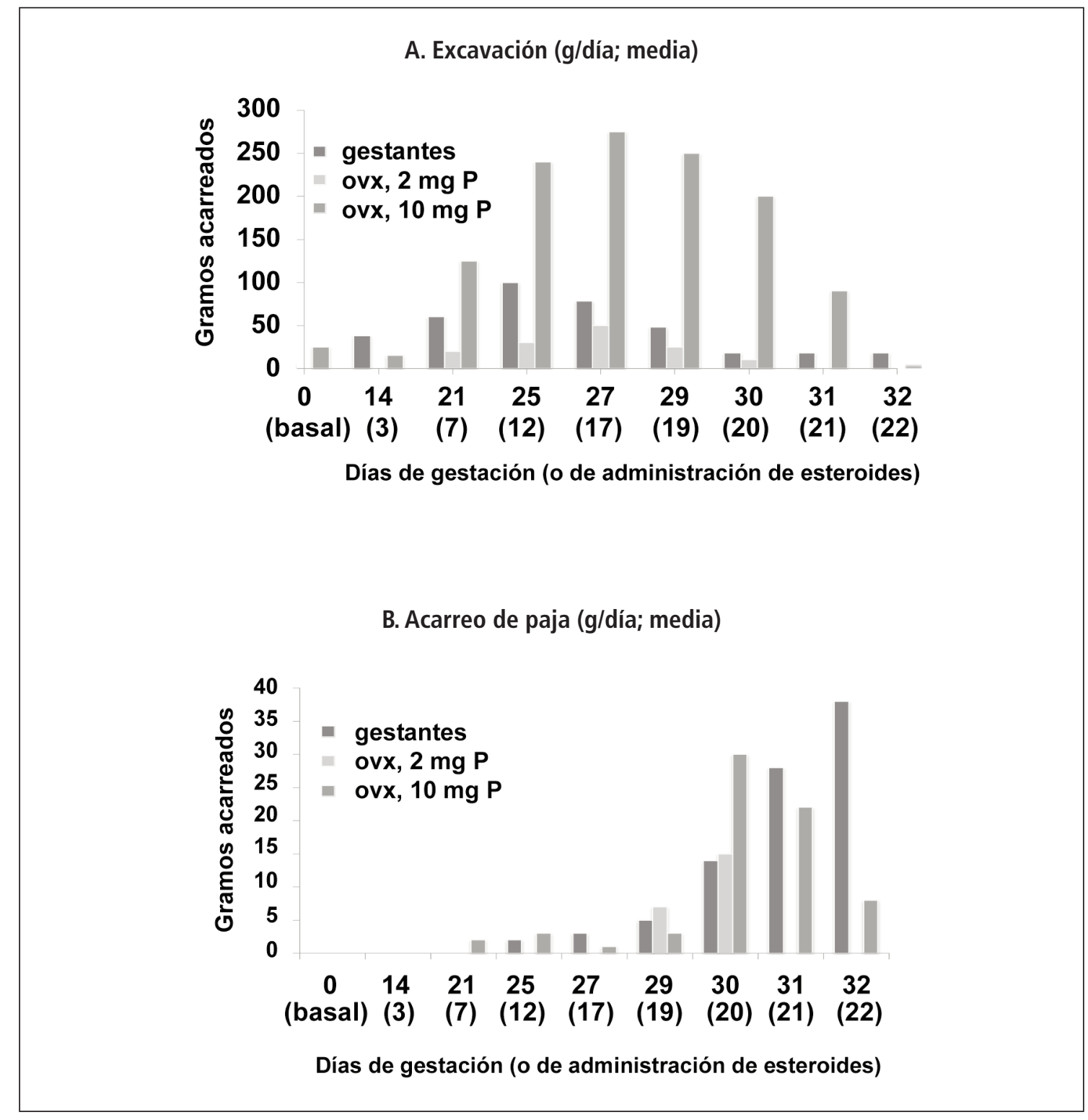

Figura 1. Variaciones en la intensidad de las conductas de excavación (panel A) y de acarreo de paja (panel B) a lo largo de la gestación (González-Mariscal et al. 1994) o durante la administración, a conejas ovariectomizadas (ovx), de benzoato de estradiol (días 1-22; $5 \mu \mathrm{g} /$ día) combinado con 2 ó $10 \mathrm{mg} /$ día de progesterona (P; días 3-16; González-Mariscal et al. 1996).

Figure 1. Variations in the intensity of digging (pannel A) and straw collection (pannel B) behaviours along gestation (González-Mariscal et al., 1994) or during administration, to ovariectomized does (ovx), of estradiol benzoate (days 1-22; $5 \mu \mathrm{g} /$ day) combined with 2 or $10 \mathrm{mg} /$ day of progesterone (P; days 3-16; González-Mariscal et al., 1996). 
centración plasmática de estas dos hormonas es breve pues, después de unos minutos, los niveles de oxitocina y prolactina regresan a niveles basales (Fuchs et al., 1984). Si bien la importancia de la prolactina y la oxitocina para la producción y evacuación láctea, respectivamente, está bien establecida, su participación en la expresión del comportamiento materno aún no está esclarecida. Si se previene la secreción de prolactina durante la primera semana de lactancia (administrando bromocriptina) se suprime la producción de leche pero no desaparece la conducta maternal. Sin embargo, al antagonizar la liberación pre-parto de prolactina por inyecciones de bromocriptina a finales de la gestación, sí se suprime el comportamiento maternal durante los primeros días de la lactancia (González-Mariscal et al., 2000). La co-inyección intracerebroventricular de prolactina restaura la conducta maternal (González-Mariscal et al., 2004) lo que indica que dicha hormona es esencial para el inicio de este comportamiento. Existe evidencia que sugiere que la oxitocina participa en la regulación de un aspecto particular de la conducta maternal: su temporalidad. Normalmente las conejas amamantan a sus crías con periodicidad circadiana, es decir, una vez cada 24 horas (Jilge, 1993 y 1995; González-Mariscal et al., 2013b). Sin embargo, si el tamaño de la camada es pequeño (menor a 6 gazapos) se pierde la periodicidad circadiana y las madres entran a la caja nido muchas veces al día (González-Mariscal et al., 2013b). De manera similar, se conoce que cada episodio de amamantamiento dura entre 3 y 5 minutos durante toda la lactancia (Zarrow et al., 1965; Lincoln, 1974; González-Mariscal et al., 1994). Sin embargo si se alteran las características normales de la succión, por ejemplo: reduciendo el número de crías que succionan (por debajo de 4; González-Mariscal et al., 2013a) o cubriendo los pezones maternos o los hocicos de las crías (González-Mariscal, 2007) se incrementa el tiempo que las madres pasan en el nido con la camada. Dado que existe una relación directa entre el número de crías amamantadas y la cantidad de oxitocina secretada (Fuchs y Wagner, 1963) hemos propuesto que esta hormona contribuye importantemente a mantener tanto la periodicidad circadiana del amamantamiento como la duración normal de los episodios de succión.

\section{Comportamiento materno, bienestar y estrés en diferentes sistemas de explotación}

Cunicultura comercial de orientación cárnica

En las granjas comerciales las conejas se alojan en jaulas individuales de varilla metálica que permiten el contacto visual, olfativo y auditivo entre vecinas (Negretti et al., $2008 \mathrm{y}$ 2010). Actualmente tienden a ser jaulas polivalentes con objeto de que los gazapos se ceben en la misma jaula en que nacieron y se reduzca así el estrés del destete, trasladando a la madre a una jaula y sala limpias para que el siguiente parto ocurra en buenas condiciones higiénico-sanitarias.

En la jaula la relación materno-filial se inicia cuando el cunicultor pone a disposición de la coneja un nidal con material apropiado (viruta, paja o borra predominantemente) 3-4 días antes del parto. Desde ese momento la hembra se ocupa de la construcción del nido, iniciando una serie de visitas al nidal que alcanzarán el máximo el día del parto y el siguiente, y se reducirán conforme aumenta la edad de los gazapos (Fernández-Carmona et al., 2005). Es raro encontrar a las conejas en el interior del nidal cuando no amamantan, y si se las ve de modo continuado, es muy probable que sufran algún problema: la pododermatitis es el más frecuente en jaulas con suelo de malla que carecen de reposapatas, ubicándose las conejas en el nidal seguramente por- 
que les conforta el suelo de madera o plástico, o el blando material. Cuando dispone de reposapatas la coneja lo utiliza muy frecuentemente, especialmente al final de la gestación (López et al., 2002), y siendo cada vez más común este objeto en las jaulas ( $75 \%$ lo tienen) se asiste paralelamente a una reducción de la morbilidad (Rosell y De la Fuente, 2013). Es una forma de enriquecimiento de la jaula y de mejora del bienestar de la hembra, pues la mortalidad y morbilidad se han definido como los dos principales indicadores del bienestar en conejos (Hoy y Verga, 2006).

Tradicionalmente la coneja tiene libre acceso al nidal, pero en muchas granjas se cierra la entrada al mismo, excepto unos minutos cada día, durante los 7-10 primeros días postparto. Este manejo imita el comportamiento de la coneja silvestre, que visita el nido una vez cada 24 horas y tapa la salida de la madriguera cuando lo abandona. Hoy sabemos que un $20-40 \%$ de las conejas entran al nido dos o tres veces al día, si bien no está comprobado que el acceso suplementario al nidal implique amamantamiento en todas las visitas. Los resultados de limitar la entrada al nido son contradictorios y mientras algunos autores lo aconsejan porque se reduce la mortalidad y el estrés de los gazapos durante la lactación (Verga et al., 2007) otros no encuentran esa ventaja (Szendrö et al., 1999; EFSA, 2005). Algunos ganaderos solamente hacen lactancia controlada 24 horas antes de la inseminación (o 48 horas con un breve amamantamiento en la mitad del periodo), utilizando esta técnica como bio-estímulo, pues la cancelación de un episodio de amamantamiento en la lactancia temprana restaura el estro en 24 horas (García-Dalmán y González-Mariscal, 2012), lo cual incrementa las probabilidades de lograr una gestación tras la inseminación (Bonanno et al., 2004).

Otro punto interesante de la mejora del bienestar en la relación materno-filial lo constituye la transferencia de gazapos de unas ca- madas a otras. Se realiza el mismo día del parto y habitualmente se iguala el número de gazapos a 9 o 10 por camada. Su fin es que los hijos de hembras altamente prolíficas puedan recibir alimentación sin estar limitados por el número de pezones de su madre, variable entre 8 y 11. En general las conejas no manifiestan rechazo de gazapos (International Rabbit Reproduction Group, 2005; González-Mariscal y Gallegos, 2007), especialmente cuando al mezclarlos se tiene la precaución de considerar tamaños similares, que la adopción sea próxima al parto y, asimismo, de mantener el nidal cerrado cierto tiempo para que los gazapos de adopción se integren en la camada. La manipulación de los gazapos también se relaciona popularmente con rechazo por parte de la madre, pero este comportamiento no se observa en las granjas. Al contrario: se ha comprobado que toquetear a los gazapos algunos minutos diarios desde su nacimiento contribuye a reducir sus reacciones de miedo frente a los humanos, por lo que se aconseja como procedimiento sencillo de mejora del bienestar (Anderson et al., 1972; Bilkó y Altbäcker, 2000; Zucca et al., 2012).

A veces en el nidal o en el suelo de la jaula puede encontrarse algún gazapo muerto mordisqueado, o restos de gazapo, habiéndose descrito en esta especie canibalismo perinatal asociado a un escaso carácter maternal debido a estrés. Es un fenómeno poco habitual en granja (Sawin y Crary, 1953) que también se observa en conejas mascota. Descartado el sacrificio con fines caníbales, parece razonable pensar que, si algún pequeño gazapo muere, quizás la coneja intenta hacer desaparecer el cadáver para mantener limpia la jaula. En otras ocasiones pueden encontrarse muertas varias camadas enteras como consecuencia de que la madre ha abandonado a los neonatos. También es inusual este abandono, y ocurre generalmente cuando coinciden los nacimientos en la nave con bajadas de temperatura externa; la colocación 
de calefactores para evitar este problema es cada vez más frecuente en las granjas.

Conforme avanza la lactación la relación materno-filial va estableciéndose en el exterior del nidal pues los gazapos empiezan a salir del mismo cuando tienen alrededor de 16-18 días de edad. Inician entonces el reconocimiento del territorio, investigan el suelo, las paredes, se acercan al comedero, al bebedero, interactúan entre sí y con la madre en múltiples ocasiones: contacto, acicalamiento, corretean, comparten comedero, suben sobre su madre para acceder al bebedero o al comedero, etc. e intentan alcanzar los pezones y son entonces rechazados por la coneja. Para la mejora del bienestar de la hembra durante este periodo se aconseja utilizar escondites u objetos que le permitan aislarse de su camada, siendo la colocación de una plataforma unos $30 \mathrm{~cm}$ por encima del suelo de la jaula la opción más estudiada, aunque aún no ha sido introducida en las granjas comerciales. Una plataforma supone aumentar la altura de la jaula de la coneja (que hoy es de $30-40 \mathrm{~cm}$ ) consiguiendo además ampliar el espacio disponible y favorecer así la realización de algunos comportamientos específicos requeridos como indicadores de bienestar -saltar, erguirse, vigilar, etc.-. Lo negativo de la plataforma es el incremento de la suciedad encima y debajo de la misma (Szendrö, 2012), y que, finalmente, los gazapos también la ocupan. Al respecto, parece que el momento de mayor concentración de intentos de lactar ocurre dos horas antes del amamantamiento en la $3^{a}$ semana de vida, y media hora antes del amamantamiento en la $5^{a}$ semana, sugiriendo Mirabito (2007) que ese comportamiento podría no ser una perturbación para las madres sino una estrategia de adaptación entre madres y gazapos que crecen.

Reproducción y cría en colonias

Como hemos indicado, las reproductoras comerciales se alojan en jaulas individuales, lo que pudiera entrar en contradicción con el carácter social de la especie, que en la naturaleza vive en colonias. Por esta razón, impulsado por las objeciones crecientes de la opinión pública y por las tendencias de algunas instancias legisladoras en contra del alojamiento individual en las condiciones de la producción ganadera predominante, se han propuesto sistemas con alojamiento de conejas reproductoras en grupo que sean implementables en el ámbito productor (Ruis, 2006). La mayoría de los prototipos han consistido en pequeños parques, de dimensiones variables en torno a 4-9 $\mathrm{m}^{2}$ en planta, con suelo emparrillado, con enriquecimientos ambientales diversos y equipados con varios nidales, en los que se alojan un macho y un número de conejas variable entre 4 y 8 , así como los gazapos hasta el destete (Stauffacher, 1992; Rommers et al., 2006; Ruis, 2006; Szendrö et al., 2013). Estos sistemas de alojamiento en colonia implican, respecto a la jaula individual, cambios en las características de los propios alojamientos y en su manejo, y plantean nuevas cuestiones relacionadas con el bienestar. Así, la libre entrada de unas conejas en los nidales de las otras, propiciada por la competencia por los lugares donde hacer el nido y por la preferencia por determinados nidales, incrementa la mortalidad de los gazapos lactantes por aplastamiento e infanticidio (Ruis, 2006). Además, en sistemas de cría en colonia se han descrito partos de dos o más conejas en el mismo nidal (Finzi y Gualterio, 1990). También ocurren agresiones entre reproductoras, particularmente cuando se introducen nuevas hembras desconocidas para el grupo, sobre todo si previamente han estado alojadas individualmente. Por otra parte, el alojamiento en colonia exige una escrupulosa higiene debido al contacto directo entre animales, siendo el control sanitario más difícil que en el alojamiento individual en jaula. Además, los sistemas de alojamiento en colonia son más complejos de manejar, dificultando el 
seguimiento de cada reproductora y su camada, la limpieza y la captura de los animales (Ruis, 2006). Otro problema importante es la alta incidencia de pseudogestaciones, que en alguna experiencia ha superado el $20 \%$ de las hembras (Rommers et al., 2006). Cuando las conejas están alojadas con el macho, el ritmo de reproducción es intensivo, con cubrición post-parto (Ruis, 2006). En un intento de racionalizar la reproducción, se ha investigado la ciclización de las conejas mediante inseminación artificial pero se ha obtenido una baja fertilidad debida en parte a la incidencia de pseudogestaciones (Rommers et al., 2006). Los intentos más exitosos de reproducción en colonia se han basado en la implementación de un sistema individual de reconocimiento electrónico del nidal, de manera que cada coneja sólo pueda acceder al suyo. Esto ha permitido mejorar algunos aspectos negativos asociados al acceso libre al nidal (se reduce la competencia por los nidales y el infanticidio), pero persisten problemas por resolver tales como el aumento del coste de producción, la elevada incidencia de pododermatitis pese a usar suelo de emparrillado plástico, las peores condiciones de higiene, la sincronización eficaz de la reproducción de las hembras y la incidencia de pseudogestaciones, o el peor crecimiento de las crías (Ruis, 2006). Además, en conejas alojadas en colonia se incrementa la cortisolemia, y por ende el estrés, respecto al alojamiento individual (Szendrö et al., 2013). Por todo ello, dicho sistema dista de convertirse en una alternativa comercialmente viable al alojamiento individual en jaula.

También se han usado y propuesto conejares más extensivos para mantener colonias en recintos sobre suelo, en condiciones próximas a las de vida libre y con poco manejo (Finzi y Mariani, 2011). Entre los sistemas que siguen actualmente este modelo destaca la producción de conejos silvestres en cercados, analizada posteriormente.
Cunicultura alternativa de carne

Como se ha dicho, cuando la coneja silvestre va a parir se aleja de la madriguera común, excava su propia hura y pare en soledad, cuidando sola su nido con total autonomía. Por esta razón, la idea de que las conejas, siendo animales sociales, tengan que alojarse en una estructura común no corresponde a la etología de la especie y explica por qué los intentos de mejorar el bienestar de las madres en espacios comunes hayan acabado en resultados negativos sea en cercados sea en jaulas colectivas. La jaula madre individual es pues, hasta ahora, la mejor solución para la fase reproductiva de las conejas desde la perspectiva etológica.

Algunas ideas equivocadas acerca del bienestar animal nacen de conceptos antropomórficos y hasta de ideas abstractas sin fundamento. Si observamos los conejos en condiciones naturales podemos notar que los animales utilizan dos condiciones ambientales totalmente diferentes (Lockley, 1964): por un lado disfrutan su sistema de madrigueras donde permanecen normalmente a lo largo del día en espacios largos pero muy estrechos, protegidos de los predadores, y por el otro lado salen al mundo exterior donde tienen necesariamente que buscar su alimento, normalmente desde la tarde hasta la madrugada, mimetizados por su pelo pardo en la oscuridad y listos para escapar a las madrigueras en presencia de predadores. Si hay condiciones de estrés, es en el pasto donde se dan situaciones de peligro y no en las estrechas y seguras galerías de las madrigueras, pues un espacio estrecho y oscuro, excavado por los propios conejos, es el que más corresponde a sus exigencias etológicas en comparación con el inseguro mundo exterior.

Además el terreno es un excelente aislante térmico natural. Por esto el interior de la madriguera es más fresco en verano y permite a los conejos sustraerse al estrés térmico siendo 
muy termosensibles, mientras que en invierno es menos fría la cavidad que tiene función de nidal para los neonatos, que, naciendo desnudos, necesitan una temperatura microambiental de $27-29^{\circ} \mathrm{C}$ para mantener su temperatura corporal por encima de $35^{\circ} \mathrm{C}$ durante los primeros días (Colin et al., 2008). Se ve entonces como el conejo disfruta en la naturaleza de condiciones microambientales que en la industria tienen que producirse con gasto de energía.

Se considera entonces que, si se logran reproducir artificialmente unas condiciones ambientales más parecidas a las naturales, se va a incrementar el bienestar de los conejos. Esto se puede conseguir de formas diferentes (Finzi y Mariani, 2011), pero el sistema más eficaz y económico, bien testado durante muchos años de actividad en pequeñas granjas, es el de celda enterrada. Está basado en una arqueta de cemento (de $50 \times 50 \times 50 \mathrm{~cm}$, de las usadas para el saneamiento de las aguas domésticas) enterrada hasta el borde y cerrada por una tapa aislante, removible para controlar el interior donde se pondrá el nidal y donde el adulto puede encontrar un microambiente más parecido a las condiciones naturales y más adaptado a sus exigencias de termorregulación. En efecto, en el interior de la celda la temperatura máxima resulta al menos seis grados inferior a la exterior en la sombra en época cálida. La celda comunica mediante un tubo con una jaula metálica estándar exterior donde se encuentran el alimento y el agua. El tubo, de $14-16 \mathrm{~cm}$ de diámetro, debe ser suficientemente largo para que el aire caliente o frío no entre en la celda $y$, al mismo tiempo, estando enterrado al menos medio metro, constituye otro enriquecimiento coherente con la etología del conejo. Los reproductores pueden escoger libremente el ambiente más confortable según sus propias exigencias fisiológicas y etológicas, lo que no puede hacer en los sistemas convencionales (Finzi y Negretti, 2007).
Una de las demandas más comunes de quienes persiguen el bienestar animal es que los animales puedan vivir en condiciones naturales. Para averiguar si los conejos prefieren, y en qué medida, un área de terreno libre con hierba y con disponibilidad de agua y pienso balanceado, en una experiencia (Finzi et al., 2001) se puso una celda enterrada en comunicación con un área de prado cercado donde había una parte de terreno llana y otra inclinada, un árbol y pienso y agua, estos últimos en condiciones iguales a las de la celda. Las hembras prefirieron claramente permanecer en la celda (Figura 2): Prácticamente salían solo para depositar sus heces al borde del cercado, lo más lejos posible de la celda, y algo más frecuentemente antes del parto sólo para excavar su madriguera en la parte inclinada del suelo. Se demuestra que las conejas escogen y prefieren el sistema artificial que cubre sus exigencias etológicas incluso mejor de lo que pueden lograr en condiciones naturales. La única exigencia etológica ancestral que manifiestan es, cuando es posible, la de excavar su madriguera para parir en el exterior.

\section{Granjas cinegéticas}

La producción de conejos silvestres en granjas cinegéticas, para repoblar cotos de caza, tiene relevancia en varios países de Europa occidental, principalmente España, Francia y Portugal (González-Redondo, 2002), y ha generado un sector productor joven pero consolidado (González-Redondo y Sánchez-Martínez, 2014). Dicha producción se basa en dos sistemas diferenciados de alojamiento y manejo de los reproductores (González-Redondo, 2002): colonias alojadas en cercados y alojamiento en jaula en sistemas análogos a los de la cunicultura convencional. En ambos casos la cría de gazapos tras el destete se realiza en cercados para habituarlos a las condiciones que encontrarán en el campo al liberarlos en la repoblación. Cada uno de estos sistemas de re- 


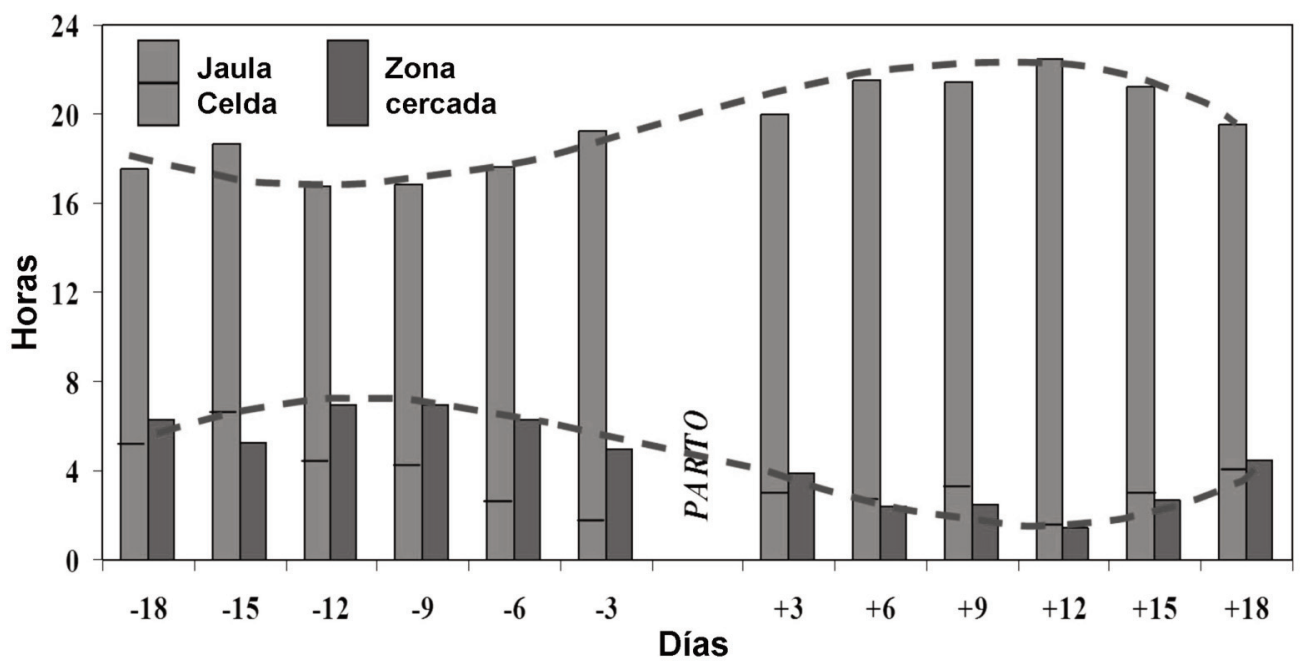

Figura 2. Tiempo de presencia de las hembras en un sistema artificial (celda o jaula) o en un cercado, con respecto al parto (Adaptado de Finzi et al., 2001).

Figure 2. Time spent by does in an artificial system (underground cell or cage) or in an enclosure, with respect to delivery date (Adapted from Finzi et al., 2001).

producción plantea distintos problemas de manejo y presenta limitaciones al bienestar. Común a ambos es el carácter silvestre del animal explotado, que incrementa la dificultad de manejo en comparación con las razas domésticas por su mayor reactividad y el mayor estrés que sufre en cautividad.

\section{Reproducción en cercados}

El alojamiento de los conejos silvestres reproductores en cercados es bien conocido desde que se utilizaron en Australia y Nueva Zelanda para estudiar, en especial a partir de la década de 1950, el funcionamiento de las colonias cunícolas como vía para mejorar el control de las plagas de esta especie invasora (Myers, 1958). Se basa en imitar las condiciones de vida naturales y consiste en alojar, en cercados de superficie muy variable (desde algún centenar de metros cuadrados hasta una hectárea o más), colonias integradas por uno o varios grupos familiares formados por un macho y 5-8 hembras cada uno, en función de la superficie del cercado (González-Redondo, 2002). Los cercados pueden dividirse en un área de alimentación y otra de reproducción comunicadas (Díez, 2013). Cuanto menor es la superficie del cercado respecto al área de campeo de la especie (que, aunque muy variable, en promedio es de 2-4 ha; Moseby et al., 2005) y mayor el tamaño de la colonia, más problemas sociales aparecen en el grupo y afectan a la reproducción. Debido a ese incremento relativo de la densidad de alojamiento respecto a las condiciones naturales, la producción de conejos silvestres en cercados está limitada cualitativamente por los mismos problemas relacionados con la reproducción que el alojamiento de conejas domésticas en grupo: agresión entre animales del mismo sexo (machos a machos, hem- 
bras a hembras, en especial las dominantes a las subordinadas) y competencia de las conejas por los lugares donde nidificar, con la consecuente pérdida de camadas por infanticidio. También son escasas las posibilidades de control reproductivo y de la cría, y mayores los riesgos sanitarios por el contacto directo entre animales y por el suelo de tierra que facilita el ciclo de las parasitosis y obliga a rotar los cercados.

La disponibilidad de lugares óptimos donde los conejos puedan excavar madrigueras afecta a la productividad de los cercados de reproducción (Guerrero-Casado et al., 2013). Por eso, un elemento esencial de estas instalaciones son los vivares artificiales donde los conejos se refugian y paren como en libertad. Su adecuado diseño y disposición puede reducir los problemas de competencia por los sitios para parir y, con ellos, las agresiones entre conejas y el infanticidio de la prole ajena. Da buen resultado la disposición de un vivar grande comunitario y de un número de pequeños vivares individuales (superficie aproximada de $1 \mathrm{~m}^{2}$ ), lo más dispersos posible dentro del cercado, que exceda ampliamente del número de conejas reproductoras (Arenas et al., 2006). La distribución en el cercado de pacas de paja sin deshacer también sirve para que sean utilizadas por algunas hembras como lugar para elaborar el nido y parir.

En estos cercados la mortalidad perinatal puede ser inferior al $17 \%$ mientras que la mortalidad acumulada al destete se sitúa en el $23 \%$ (Arenas et al., 2006), lo que revela la viabilidad del sistema para la cría de conejos silvestres para repoblación.

Un problema son, al igual que el alojamiento de conejas domésticas en colonia, las agresiones que sufren los animales ajenos a la colonia cuando son introducidos para reposición, que llegan incluso a morir por las agresiones de los residentes (Cortés, com. pers.). Este problema se evita en parte dejando como animales de reposición conejos nacidos en la propia colonia (Arenas et al., 2006), lo cual, a su vez, obliga a una buena gestión del plantel reproductor.

\section{Reproducción en jaulas}

En un intento de mejorar el control reproductivo, y aprovechando los equipamientos y tecnología para la producción del conejo doméstico, muchas granjas cinegéticas alojan los reproductores silvestres en jaulas comerciales de conejo de carne (González-Redondo, 2002; González-Redondo y Sánchez-Martínez, 2014). Dada la naturaleza salvaje, y por ello reactiva, del conejo silvestre, este sistema de alojamiento plantea condicionantes que limitan el bienestar y empeoran los resultados obtenidos en la reproducción y en la cría.

Por una parte, los conejos silvestres experimentan miedo ante la presencia del cunicultor pues si tienen un refugio a disposición, lo usan permanentemente en presencia de personas (González-Redondo, 2006). Por eso, proporcionarles algún tipo de refugio en la jaula es imprescindible para mejorar su bienestar.

Otra consecuencia del miedo experimentado por los conejos silvestres en jaula es que los animales rehúsan aparearse en presencia del cunicultor, e incluso en ocasiones la cópula no tiene lugar hasta pasado algún día desde la introducción de la coneja en la jaula del macho, posiblemente debido al estrés por el cambio de jaula (González-Redondo, 2002). Esto complica el manejo en la monta natural al ser difícil constatar las cubriciones, y es uno de los motivos por los que algunas granjas cinegéticas practican la inseminación artificial (Dávila et al., 2004; González-Redondo y Sánchez-Martínez, 2014). Indicador indirecto del estrés que padecen los conejos silvestres en cautividad estricta es que una parte (hasta un tercio) de los individuos de reposición, tanto machos como hembras, no llegan a reproducirse nunca en jaula (González-Redondo, 2002), lo que, a su vez, obliga a aumentar la tasa de reposición. 
Al igual que a la coneja doméstica, a la silvestre alojada en jaula se le proporciona un nidal para parir. Éste puede ser un modelo usado en cunicultura de carne. Pero se ha propuesto un prototipo (González-Redondo, 2006) consistente en un receptáculo cerrado, de pequeñas dimensiones (cámara interior con planta de $17 \times 23 \mathrm{~cm}^{2}$ y $20 \mathrm{~cm}$ de altura), con un tubo estrecho de entrada (luz de $9 \times 8 \mathrm{~cm}^{2}$ ) y con tapa para el registro del interior, donde se coloca una bandeja-cuna para acoger el nido. Este diseño se adapta a las exigencias etológicas y tamaño corporal del conejo silvestre por asemejarse en forma y tamaño a una hura de parto natural. A diferencia de la cunicultura de carne, en la que el nidal se proporciona a los 28 días de la cubrición, en el caso del conejo silvestre la disponibilidad permanente del modelo descrito permite a las conejas utilizarlo también como refugio, lo que puede reducir sus niveles de estrés y contribuye a mejorar la eficiencia reproductiva en jaula.

El conjunto de eventos que caracterizan la conducta materna de la coneja silvestre en jaula, en el parto y la lactancia, es similar al de la doméstica. Pero su naturaleza más reactiva evidencia peculiaridades que indican pérdida de bienestar, tanto de la coneja como de sus crías, y reducción de la productividad. Se han descrito mortalidades perinatales del $33 \%$ y durante la lactancia del $16 \%$ de los nacidos vivos, que reducen la productividad potencial desde los 3,2 gazapos nacidos en total a 2 gazapos destetados por parto, y que están originadas por el fracaso de la conducta maternal en el periparto (González-Redondo, 2010). Así, sólo el 71\% de las conejas forraban el nidal con pelo, sólo el $58 \%$ introducían paja en el mismo, en casi un $19 \%$ de las camadas al menos un gazapo era parido fuera del nidal (valor mucho más alto que en conejas domésticas; Sawin y Crary, 1953); y en un $13 \%$ de las mismas al menos un gazapo era canibalizado, incidencia también mayor en la coneja silvestre en jaula que puede asociarse al estrés que experimenta (González-Redondo y Zamora-Lozano, 2008). Este fracaso de la conducta maternal en el periparto está correlacionado con la mayor mortalidad perinatal, canibalismo y partos fuera del nidal que se observan cuando la coneja silvestre falla en elaborar el nido; es decir, cuando no introduce paja en el nidal y cuando no lo forra con su pelo, fallos que manifiestan en mayor medida las primíparas, aunque también se da en multíparas (González-Redondo, 2010). Por el contrario, las camadas de las conejas silvestres que elaboran correctamente los nidos sobreviven al destete. Por otra parte, muchas conejas silvestres intentan tapar con paja la boca del nidal como lo hacen con la entrada de la hura de parto en libertad, de ahí la importancia de un adecuado diseño del nidal y de aprovisionarlas abundantemente con paja para que puedan expresar esta conducta.

\section{Situación e implicaciones de la legislación sobre bienestar animal}

Hasta hace muy poco, en ningún país existía legislación relativa al bienestar de los conejos en producción. A partir de la década de 1980, países como Reino Unido, Canadá o Australia fueron pioneros en la promulgación de códigos de recomendaciones o de buenas prácticas específicos para salvaguardar el bienestar en el manejo intensivo del conejo doméstico en producción comercial, sobre las premisas de un buen cuidado por parte del cunicultor, de la provisión de alojamiento adecuado y de permitir el desarrollo de las necesidades etológicas y fisiológicas esenciales de la especie (DEFRA, 1987; SCARM, 1991; Villagrá et al., 2012). Así, en Reino Unido se estableció la necesidad de proporcionar al menos $5600 \mathrm{~cm}^{2}$ de superficie de suelo de la jaula para una coneja con su camada de hasta cinco semanas de edad, ampliada a $7400 \mathrm{~cm}^{2}$ cuando los ga- 
zapos permanecían hasta las ocho semanas de edad con la madre. Para el nidal establecía unas dimensiones mínimas de $30 \mathrm{~cm}$ de longitud y una superficie en planta de al menos $800 \mathrm{~cm}^{2}$ que permitiese a las conejas un adecuado amamantamiento de la camada, y que debía construirse de manera que permitiese el fácil acceso de la coneja pero una retención adecuada de la camada en su interior para que los gazapos no perezcan de frío si salen accidentalmente del mismo. Recomendaban cubrir los nidales abiertos durante el parto para propiciar un ambiente oscuro y protegido similar al de una madriguera, así como perturbar lo mínimo las camadas con menos de una semana y no destetar antes de las cuatro semanas de edad (DEFRA, 1987). En Australia se mantuvieron las necesidades de espacio en las jaulas propuestas en el código británico y se introdujo la recomendación de proporcionar el nidal con material de cama al menos con dos días de antelación a la fecha prevista de parto, para que la coneja pueda desarrollar adecuadamente la conducta de elaboración del nido (SCARM, 1991).

En 1998 el Consejo de Europa inició los intentos de elaboración de unas recomendaciones (base de una posible legislación) relativas a la protección del bienestar de los conejos pero, debido a las diferentes posturas de los Estados miembros, no se ha alcanzado consenso, pese a haber desarrollado hasta 18 borradores de las mismas (Villagrá et al., 2012). Durante ese proceso de elaboración de las mencionadas recomendaciones, la Agencia Europea de Seguridad Alimentaria publicó en 2005 un documento que recoge la opinión científica de un panel independiente de expertos sobre el impacto de los sistemas actuales de alojamiento y manejo sobre la salud y bienestar de los conejos domésticos de granja (EFSA, 2005), que revisa las evidencias científicas al respecto. En lo relativo al comportamiento maternal de la coneja y su camada, destacó que la tecnología de alojamiento de hembras reproductoras en grupo no se encontraba lo suficientemente desarrollada como para poder recomendar su implementación viable a escala de granja comercial, y de hecho está siendo ampliamente estudiada en los últimos años. En lo referente a las jaulas individuales para conejas reproductoras, se establece que deben tener anchura suficiente para permitirles girarse cómodamente (al menos $38 \mathrm{~cm}$ ) y suficiente longitud para que la coneja pueda tenderse con las patas extendidas (entre 65 y $75 \mathrm{~cm}$ ), teniendo a su disposición al menos $3500 \mathrm{~cm}^{2}$. Se señala también la necesidad de que la hembra cuente con un nidal cerrado en el que poder elaborar un nido y parir (de forma similar a lo indicado por DEFRA, 1987), indicándose que la superficie del nidal no debe computarse en la superficie minima del suelo de la jaula que debe tener la reproductora a su disposición. Se concluye también que el tamaño de los nidales actualmente utilizados parece ser adecuado para satisfacer las necesidades etológicas de la coneja y su camada (EFSA, 2005).

En este contexto, en los últimos años las demandas de una regulación de las condiciones de alojamiento y cría han ido aumentando, hasta que países como Bélgica, Reino Unido, Suiza, Holanda, Austria o Alemania han desarrollado su propia legislación al respecto. En todos ellos, un criterio que ha primado sobre el resto es el de permitir a los conejos desarrollar comportamientos naturales, tanto en el caso de los gazapos de cebo como en las reproductoras, pero también criterios de higiene, ambiente y bienestar en general (Hoy, 2008). Paradójicamente, los tres principales países europeos productores de carne de conejo, que son Italia, España y Francia, aún no han promulgado legislación específica para la salvaguarda del bienestar de la especie (Rafel et al., 2013).

La tendencia principal es a aumentar el espacio de las jaulas $y$, en los países europeos con legislaciones propias, a alojar a las conejas reproductoras en grupo. Los espacios requeridos para alojamientos individuales de 
conejas oscilan entre los $3600 \mathrm{~cm}^{2} /$ coneja propuestos por el Comité Permanente del Consejo de Europa para la Protección de los Animales de Granja (Hoy y Verga, 2006) y los $5600 \mathrm{~cm}^{2} /$ coneja ya mencionados del Reino Unido (Alfonso, 2013; Tabla 2).
Existen también algunos aspectos controvertidos en lo relativo a espacios, como que los animales se puedan levantar con las orejas extendidas (posición de alerta o exploración), que se permitirían en jaulas más altas, y con plataformas elevadas (Hansen y Berthelsen, 2000).

Tabla 2. Superficies y requerimientos de las jaulas individuales en los países con legislación propia en bienestar cunícola

Table 2. Area and requirements of individual cages in countries with own regulations on rabbit welfare

\begin{tabular}{llcr}
\hline País & Espacio madres y camada hasta 5 semanas & Plataforma & Altura \\
\hline Reino Unido & $5600 \mathrm{~cm}^{2}$ & No especifica & $45 \mathrm{~cm}$ \\
Holanda & $4500 \mathrm{~cm}^{2}$ & $900 \mathrm{~cm}^{2}$ & $60 \mathrm{~cm}$ \\
Alemania & $4000 / 4800 \mathrm{~cm}^{2}$ & Opcional & $40 / 60 \mathrm{~cm}$ \\
& (con o sin plataforma) para $>5,5 \mathrm{~kg}$ & $1000 \mathrm{~cm}^{2}$ & \\
Austria & Jaulas prohibidas & & \\
Bélgica & Jaulas prohibidas a partir de 2021 (plan paso a paso) & & \\
Suiza & Jaulas prohibidas & & \\
\hline
\end{tabular}

Asimismo, otros aspectos contemplados en las legislaciones y códigos de recomendaciones, son los relacionados con el enriquecimiento ambiental. Así, se ha propuesto usar refugios (Hawkins et al., 2008) debido a que la naturaleza de presas de estos animales en estado silvestre hace que se mantengan fuertes respuestas de miedo (Baumans, 2005). Por otro lado, la posibilidad de realizar un nido se considera indiscutible en cualquier sistema productivo.

En cuanto a los sistemas de alojamiento en grupo que se están proponiendo en las legislaciones nacionales existentes, facilitan los contactos sociales entre las conejas reproductoras. Sin embargo, cabe destacar dos aspectos: el primero, que en condiciones naturales las conejas se aíslan para parir, lo cual debe tenerse en cuenta a la hora de diseñar los alojamientos, y por otro lado, los proble- mas de agresividad entre las reproductoras que existen a día de hoy y deben ser solucionados (Rommers y Kemp, 2012). Además, estos sistemas también pueden ser cuestionados por aspectos relacionados con la higiene (EFSA, 2005), sobre todo en sistemas en suelo, aunque cada vez más se tiende a sistemas enrejillados.

Además, es importante no olvidar las implicaciones económicas que pudieran tener estos cambios legislativos. En el caso del aumento de la superficie por coneja, se reduce el número de reproductoras en el mismo espacio, y además podría implicar unos mayores costes (Bignon et al., 2012), mientras que en los alojamientos en grupo los problemas de agresividad podrían provocar una disminución del rendimiento productivo de las granjas (Szendrö et al., 2013). 


\section{Conclusiones}

La coneja doméstica conserva buena parte del repertorio etológico silvestre relativo a la reproducción y cuidado materno, de fina regulación endocrina, tal vez por lo tardío de la domesticación y la intensificación de la cunicultura basada en el alojamiento individual de los reproductores en jaula. Dicha intensificación comporta algunas limitaciones a la expresión de la conducta silvestre y situaciones de pérdida de bienestar, que se han intentado resolver mediante el alojamiento de las conejas en colonia, sin éxito efectivo como alternativa comercialmente viable al alojamiento individual en jaula. Pese a ello, hay una tendencia a que los marcos normativos sobre alojamiento proporcionen a las conejas más espacio y enriquecimiento ambiental y en algunos casos propongan suprimir las jaulas convencionales. Otros sistemas alternativos presentan peculiaridades relativas a la expresión de la conducta materna y el bienestar. Así, la producción cinegética acarrea mayor fracaso de la conducta materna por el estrés causado por la cautividad, perdiéndose bienestar y productividad. Otros sistemas, como la cría alternativa al aire libre en celdas enterradas, han resuelto problemas como la incidencia negativa del estrés térmico sobre la reproducción.

\section{Bibliografía}

Alfonso C (2013). ¿Qué está pasando en otros países de Europa sobre el Bienestar Animal? Jornada Bienestar ASESCU "¿Hacia dónde debe ir el bienestar de los conejos de granja en España?". Zaragoza, 21 marzo: 18 pp.

Anderson CO, Denenberg VH, Zarrow MX (1972). Effects of handling and social isolation upon the rabbit's behaviour. Behaviour 43: 165-175.

Arenas AJ, Astorga RJ, García I, Varo A, Huerta B, Carbonero A, Cadenas R, Perea A (2006). Cap- tive breeding of wild rabbits: Techniques and population dynamics. Journal of Wildlife Management 70: 1801-1804.

Baumans V (2005). Environmental enrichment for laboratory rodents and rabbits: requirements of rodents, rabbits, and research. ILAR Journal 46: 162-170.

Bignon L, Bouchier M, Coutelet G, Galliot P, Souchet C, Fortun-Lamothe L (2012). Individual housing of young does in different sized cages: impact on welfare, economic costs and productive data. 10 $0^{\text {th }}$ World Rabbit Congress. Sharm El Sheikh, 3-6 Septiembre. 1045-1049.

Bilkó Á, Altbäcker V (2000). Regular handling early in the nursing period eliminates fear responses toward human beings in wild and domestic rabbits. Developmental Psychobiology 36: 78-87.

Bonanno A, Mazza F, Di Grigoli A, Alabiso M (2004). Effects of a split 48-hour doe-litter separation on productivity of free-nursing does and their litters. Livestock Production Science 89: 287-295.

Broekhuizen S, Bouman E, Went W (1986). Variation in timing of nursing in the Brown Hare (Lepus europaeus) and the European Rabbit (Oryctolagus cuniculus). Mammal Review 16: 139-144.

Caba M, González-Mariscal G (2009). The rabbit pup, a natural model of nursing anticipatory activity. European Journal of Neuroscience 30: 1697-1706.

Canali E, Ferrante V, Todeschini R, Verga M, Carenzi $C$ (1991). Rabbit nest construction and its relationship with litter development. Applied Animal Behaviour Science 31: 259-266.

Colin M, Fiorenza G, Balogh K, Virág G (2008). Cooling panel type and location influence nest temperature, growth and sanitary status of rabbits during the cold season in Hungary. $9^{\text {th }}$ World Rabbit Congress. Verona, 10-13 junio. 929-933.

Dávila M, Badía S, Rebollar PG (2004). Primeros resultados de inseminación artificial en conejas de monte en cautividad. XXIX Symposium de Cunicultura de ASESCU. Lugo, 31 marzo-1 abril. 113-118. 
De Blas C, Gutiérrez I, Carabaño R (1999). Destete precoz en gazapos. Situación actual y perspectivas. XV Curso de Especialización FEDNA. Madrid. 67-81.

DEFRA (1987). Codes of recommendations for the welfare of livestock: Rabbits. http://adlib.every site.co.uk/adlib/defra/content.aspx?id=000HK27 7ZX.0CORE72PLFWJLA [Acceso: 8 de enero de 2015].

Deutsch JA (1957). Nest building behaviour of domestic rabbits under semi-natural conditions. The British Journal of Animal Behaviour 5: 53-54.

Díez C, Sánchez-García C, Pérez JA, Bartolomé DJ, González V, Wheatley CJ, Alonso ME, Gaudioso VR (2013). Behavioural activity of wild rabbits (Oryctolagus cuniculus) under semi-natural rearing systems: establishing a seasonal pattern. World Rabbit Science 21: 263-370.

EFSA (2005). Scientific report: The Impact of the current housing and husbandry systems on the health and welfare of farmed domestic rabbits. Anexo a EFSA Journal 267: 1-137.

Fernández-Carmona J, Solar A, Pascual JJ, Blas E, Cervera C (2005). The behaviour of farm rabbit does around parturition and during lactation. World Rabbit Science 13: 253-277.

Finzi A, Ciorba P, Macchioni P (2001). Evaluación comparada del bienestar del conejo en sistemas alternativos de cría. XXVI Symposium de Cunicultura de ASESCU-I Symposium Ibérico de Cunicultura APEZ-ASPOC. Aveiro, 23-24 mayo. 164-167.

Finzi A, Gualterio L (1990). Nidi in comune nell'etologia cunicola. Rivista di Coniglicoltura 27(3): 29-31.

Finzi A, Mariani G (2011). L'allevamento ecologico del coniglio. Italia. Edagricole. 130 pp.

Finzi A, Negretti P (2007). Un sistema alternativo di allevamento per il benessere e la sanità del coniglio. Giornate di Coniglicoltura ASIC 2007. Forlì, 26-27 septiembre. 41-54.

Fuchs AR, Cubile L, Dawood MY, Jørgensen FS (1984). Release of oxytocin and prolactin by suckling rabbits throughout lactation. Endocrinology 114: 462-469.
Fuchs AR, Wagner G (1963). Quantitative aspects of the release of oxytocin by suckling in unanaesthetized rabbits. Acta Endocrinologica 44: 581-592.

García-Dalmán C, González-Mariscal G (2012). Major role of suckling stimulation for inhibition of estrous behaviors in lactating rabbits: acute and chronic effects. Hormones and Behavior 61: 108-113.

Gibb JA (1993). Sociality, time and space in a sparse population of rabbits (Oryctolagus $\mathrm{Cu}$ niculus). Journal of Zoology 229: 581-607.

Gómez-Conde MS, Chamorro S, Nicodemus N, De Blas C, García J, Carabaño R (2004). Efecto del tipo de fibra en la alimentación de gazapos destetados precozmente. XXIX Symposium de Cunicultura de ASESCU. Lugo, 31 marzo-1 abril. 157-163.

González-Mariscal G (2007). Mother rabbits and their offspring: timing is everything. Developmental Psychobiology 49: 71-76.

González-Mariscal G, Chirino R, Flores-Alonso JC, Rosenblatt JS, Beyer C (2004). Intracerebroventricular injections of prolactin counteract the antagonistic effect of bromocriptine on rabbit maternal behaviour. Journal of Neuroendocrinology 16: 949-955.

González-Mariscal G, Chirino R, Rosenblatt JS, Beyer C (2005). Forebrain implants of estradiol stimulate maternal nest-building in ovariectomized rabbits. Hormones and Behavior 47: 272-279.

González-Mariscal G, Díaz-Sánchez V, Melo Al, Beyer C, Rosenblatt JS (1994). Maternal behavior in New Zealand white rabbits: quantification of somatic events, motor patterns and steroid plasma levels. Physiology \& Behavior 55: 1081-1089.

González-Mariscal G, Gallegos JA (2007). New Zealand white rabbits show non-selective nursing in various types of nests. World Rabbit Science 15: 167-172.

González-Mariscal G, Lemus AC, Vega-Gonzalez A, Aguilar-Roblero R (2013b). Litter size determines circadian periodicity of nursing in rabbits. Chronobiology International 30: 711-718. 
González-Mariscal G, Melo Al, Jiménez P, Beyer C, Rosenblatt JS (1996). Estradiol, progesterone, and prolactin regulate maternal nest-building in rabbits. Journal of Neuroendocrinology 8: 901-907.

González-Mariscal G, Melo Al, Parlow AF, Beyer C, Rosenblatt JS (2000). Pharmacological evidence that prolactin acts from late gestation to promote maternal behaviour in rabbits. Journal of Neuroendocrinology 12: 983-992.

González-Mariscal G, Toribio A, Gallegos JA, Serrano-Meneses MA (2013a). The characteristics of suckling stimulation determine the daily duration of mother-young contact and milk output in rabbits. Developmental Psychobiology 55: 809-817.

González-Redondo P (2002). Producción del conejo silvestre en cautividad. II Jornadas Internacionais de Cunicultura. Vila Real, 11-12 octubre. 111-128.

González-Redondo P (2006). Proposal of a nest box for the reproduction of wild rabbits (Oryctolagus cuniculus) in cages. World Rabbit Science 14: 115-121.

González-Redondo P (2010). Maternal behaviour in the peripartum influences preweaning kit mortality in cage-bred wild rabbits. World Rabbit Science 18: 91-102.

González-Redondo P, Sánchez-Martínez R (2014). Characterisation of wild rabbit commercial game farms in Spain. World Rabbit Science 22: 51-58.

González-Redondo P, Zamora-Lozano M (2008). Neonatal cannibalism in cage-bred wild rabbits (Oryctolagus cuniculus). Archivos de Medicina Veterinaria 40: 281-287.

Guerrero-Casado J, Ruiz-Aizpurua L, Carpio AJ, Tortosa FS (2013). Factors affecting wild rabbit production in extensive breeding enclosures: how can we optimise efforts? World Rabbit Science 21: 193-199.

Hansen LT, Berthelsen H (2000). The effect of environmental enrichment on the behaviour of caged rabbits (Oryctolagus cuniculus). Appled Animal Behaviour Science 68: 163-178.
Hawkins P, Hubrecht R, Buckwell A, Cubitt S, Howard B, Jackson A, Poirier GM (2008). Refining rabbit care. UK. RSPCA-UFAW.

Hoy S, Seitz K, Selzer D, Schüddemage M (2000). Nursing behaviour of domesticated and wild rabbit does under different keeping conditions. $7^{\text {th }}$ World Rabbit Congress. Valencia, 4-7 julio. 537-543.

Hoy S, Verga M (2006). Welfare indicators. En: Maertens L, Coudert P. Recent advances in rabbit sciences. Melle, Bélgica. ILVO. 71-74.

Hoy S (2008). Guidelines for minimum standards on rabbit housing in Germany. $9^{\text {th }}$ World Rabbit Congress. Verona, 10-13 Junio. 1183-1187.

Hudson R, Distel H (1982). The pattern of behaviour of rabbit pups in the nest. Behaviour 79: 255-271.

Hudson R, Schaal B, Bilko Á, Altbäcker V (1996). Just three minutes a day: the behaviour of young rabbits viewed in the context of limited maternal care. $6^{\text {th }}$ World Rabbit Congress. Toulouse, 9-12 julio. 2: 395-403.

International Rabbit Reproduction Group (2005). Recommendations and guidelines for applied reproduction trials with rabbit does. World Rabbit Science 13: 147-164.

Jilge B (1993). The ontogeny of circadian rhythms in the rabbit. Journal of Biological Rhythms 8: 247-260.

Jilge B (1995). Ontogeny of the rabbit's circadian rhythms without an external zeitgeber. Physiology \& Behavior 58: 131-140.

Kolb HH (1991). Use of burrows and movements of wild rabbits (Oryctolagus cuniculus) in an area of hill grazing and forestry. Journal of Applied Ecology 28: 892-905.

Lebas F, Coudert P, De Rochambeau H, Thébault RG (1996). El conejo. Cría y patología. Roma, Italia. FAO. 227 pp.

Lincoln W (1974). Suckling: a time constant in the nursing behavior of the rabbit. Physiology \& Behavior 13: 711-714.

Lloyd HG, McCowan D (1968). Some observations on the breeding burrows of the wild rabbits Oryctolagus cuniculus on the island of Skokholm. Journal of Zoology 156: 540-549. 
Lockley RM (1961). Social structure and stress in the rabbit warren. Journal of Animal Ecology 30: 385-423.

Lockley RM (1964). The private life of the Rabbit. Andre Deutsch Ltd. Londres. 152 pp.

López M, María G, Paniagua P (2002). The effect of cage size on the behavior of the reproductive doe. En: COST Action 848 - $2^{\text {nd }}$ Meeting of Workgroup 2, Stuttgart, Hohenheim, 15-16 marzo. 10 pp.

Matics Z, Gerencsér Z, Mikó A, Radnai I, Odermatt M, Nagy I, Szendrö Z (2012). Effect of different lighting schedules (16L:8D or 12L:6D) on nursing behaviour of rabbit does. 10th World Rabbit Congress. Sharm El-Sheikh, 3-6 septiembre. 1063-1067.

McNeilly AS, Friesen HG (1978). Prolactin during pregnancy and lactation in the rabbit. Endocrinology 102: 1548-1554.

Mirabito L (2007). Logement et bien-être du lapin: plus de questions que de réponses? INRA Productions Animales 20: 59-64.

Morgado E, Juárez C, Melo Al, Domínguez B, Lehman MN, Escobar C, Meza E, Caba M (2011). Artificial feeding sychronizes behavioral, hormonal, metabolic and neural parameters in mother-deprived neonatal rabbit pups. European Journal of Neuroscience 34: 1807-1816.

Moseby KE, De Jong S, Munro N, Piek A (2005). Home range, activity and habitat use of European rabbits (Oryctolagus cuniculus) in arid Australia: implications for control. Wildlife Research 32: 305-311.

Myers K (1958). Further observations on the use of field enclosures for the study of the wild rabbit, Oryctolagus cuniculus (L). CSIRO Wildlife Research 3: 40-49.

Negretti P, Bianconi G, Finzi A (2008). Mutual visual relationships of rabbits raised in individual cages. $9^{\text {th }}$ World Rabbit Congress. Verona, 1013 Junio. 1213-1216.

Negretti P, Bianconi G, Finzi A (2010). Short communication: Mutual olfactory relationships in rabbits raised in individual cages. World Rabbit Science 18: 33-36.
Rafel O, Valls R, Ramon J (2013). Bienestar animal en conejos. Situación legislativa. Cunicultura 224: 13-17.

Rommers JM, Boiti C, De Jong I, Brecchia G (2006). Performance and behaviour of rabbit does in a group-housing system with natural mating or artificial insemination. Reproduction Nutrition Development 46: 677-687.

Rommers JM, Kemp B (2012). Effect of grouphousing of young does during rearing on reproduction performance and aggression: a pilot study. $10^{\text {th }}$ World Rabbit Congress. Sharm El Sheikh, 3-6 Septiembre. 1101-1105.

Rosell JM, de la Fuente LF (2013). Assessing ulcerative pododermatitis of breeding rabbits. Animals 3: 318-326.

Ross S, Denenberg VH, Sawin PB, Meyer P. (1956). Changes in nest-building behaviour in multiparous rabbits. British Journal of Animal Behaviour 4: 69-74.

Ruis M (2006). Group housing of breeding does. En: Maertens L, Coudert P. Recent advances in rabbit sciences. Melle, Bélgica. ILVO. 99-105.

Sawin PB, Crary DD (1953). Genetic and physiological background of reproduction in the rabbit: II. Some racial differences in the pattern of maternal behavior. Behaviour 6: 128-146.

SCARM (1991). Model code of practice for the welfare of animal. Intensive husbandry of rabbits. Collingwood, Australia. CSIRO Publishing.

Schaal B, Coureaud G, Langlois D, Giniès C, Sémon E, Perrier G (2003). Chemical and behavioural characterization of the rabbit mammary pheromone. Nature 424: 68-72.

Stauffacher M (1992). Group housing and enrichment cages for breeding, fattening and laboratory rabbits. Animal Welfare 1: 105-125.

Szendrö Z, Kustos K, El-Din SS (1988). Hair pull of rabbits does and its relationship with their rearing ability. $4^{\text {th }}$ World Rabbit Congress. Budapest, 10-14 octubre. vol. III: 173-181.

Szendrö Z (2012). New perspectives of housing reproducting and growing rabbits. $10^{\text {th }}$ World Rabbit Congress. Sharm El-Sheikh, 3-6 septiembre. 979-996. 
Szendrö Zs, Mikó A, Odermatt M, Gerencsér Zs, Radnai I, Dezséry B, Garai É, Nagy I, Szendrö K, Matics Z (2013). Comparison of performance and welfare of single-caged and group-housed rabbit does. Animal 7: 463-468.

Szendrö Zs, Radnai I, Theau-Clément M, Jovánczai Zs, Biró-Németh E, Milisits G, Poigner J (1999). Some factors influencing the effectiveness of post partum artificial insemination. 11 $11^{\text {th }}$ Hungarian Conference on Rabbit Production. Kaposvár, 26 mayo. 113-118.

Vega MD, Barrio M, Quintela LA, Becerra JJ, Cainzos J, Prieto A, Rodríguez-Zamora A, Herradón PG (2012). Evolución del manejo reproductivo en cunicultura. ITEA-Información Técnica Económica Agraria 108: 172-190.

Verga M, Leone P, Soliano S, Carenzi C, Crimella C (1984). Indagine su alcuni materiali alternativi per la preparazione del nido nella specie cunicola. $3^{\text {rd }}$ World Rabbit Congress. Roma, 4-8 abril. vol. Il:156-165.

Verga M, Luzi F, Carenzi C (2007). Effects of husbandry and management systems on physio- logy and behaviour of farmed and laboratory rabbits. Hormones \& Behavior 52: 122-129.

Villagrá A, Olivas I, Estellés F, Blas E, Rodríguez T, Rosell J, Pascual JJ (2012). How far rabbit cage's space recommendations reach: the gap between science and regulations. $10^{\text {th }}$ World Rabbit Congress. Sharm El-Sheikh, 3-6 septiembre. 1057-1061.

Xiccato G, Trocino A, Sartori A, Queaque PI (2004). Destete precoz del conejo: efecto de la edad y del peso al destete y del nivel de grasa del alimento. XXIX Symposium de Cunicultura de ASESCU. Lugo, 31 marzo-1 abril. 151-156.

Zarrow MX, Denenberg VH, Anderson CO (1965). Rabbit: frequency of suckling in the pup. Science 150: 1835-1836.

Zucca D, Redaelli V, Marelli SP, Bonazza V, Heinzl E, Verga M, Luzi F (2012). Effect of handling in pre-weaning rabbits. World Rabbit Science 20: 97-101.

(Aceptado para publicación el 3 de marzo de 2015) 